\title{
Diversidad, distribución, riqueza y abundancia de condrictios de aguas profundas a través del archipiélago patagónico austral, Cabo de Hornos, Islas Diego Ramírez y el sector norte del paso Drake
}

\author{
Diversity, distribution, richness and abundance of deep-sea Chondrichthyans along south Patagonian \\ Archipelago, Cape Horn, Diego Ramirez Islands and the northern area of the Drake passage \\ Pablo R. Reyes ${ }^{1}$ y Juan P. Torres-Florez ${ }^{2}$ \\ ${ }^{1}$ Ingendesa. Grupo de Levantamiento de Líneas de Base, Subgerencia de Medio Ambiente, Santa Rosa 79, Piso 9, Santiago, Chile \\ ${ }^{2}$ Instituto de Ecología y Evolución, Universidad Austral de Chile, Casilla 567, Valdivia, Chile \\ prrlt@ingendesa.cl
}

\begin{abstract}
This study describes the diversity, distribution, richness, abundance and species composition of the deep sea Chondrichthyes (1,000-2,250 m) of the south Patagonian Archipelago, including Cape Horn, Diego Ramirez Islands and the northern area of the Drake passage, south eastern Pacific Ocean ( $\left.52^{\circ} 43^{\prime} \mathrm{S}-57^{\circ} 05^{\prime} \mathrm{S}\right)$. The chondrichthyan fauna analyzed corresponds to the southernmost not Antarctic of the planet and consisted of 7 species belonging to 3 orders and 5 families. The species are the skates Amblyraja amblyraja frerichsi, Bathyraja cousseauae, Bathyraja meridionalis y Bathyraja macloviana and the sharks Somniosus pacificus, Squalus acanthias and Lamna nasus. The highest species richness was represented by the order Rajiformes, with 3 species of Arhynchobatidae and one species of the Rajidae, followed by
\end{abstract}

the order Squaliformes, with one species of Squalidae and one species of Somniosidae. The order Lamniformes was represented by Lamnidae. The biomass was dominated by Rajidae and secondly by Somniosidae. Diego Ramirez Islands had the highest abundance and species richness, Desolation Island presented the greatest diversity and equity. The chondrichthyan fauna detected in deep waters of the southern area of the southeastern Pacific shows a strong similarity with South West Atlantic waters, even more than the one existing at mid-latitudes of the South Eastern Pacific, which corroborates the biogeographic patterns observed for coastal fish in the same area, for deepwater chondrichthyans.

Key words: sharks, skates, patagonian toothfish, Dissostichus eleginoides, by-catch and discard, Chilean Patagonia

\section{Introducción}

La costa del Pacífico sur oriental está dividida en dos sectores físicos con características particulares. Desde Ecuador $\left(0^{\circ} \mathrm{S}\right)$ hasta la parte norte de la isla de Chiloé ( $41^{\circ} 45^{\prime}$ S), la línea de costa es continua. Desde Chiloé hasta el Cabo de Hornos (55⒌'S) es muy fragmentada, con el sistema de fiordos, canales e islas más extenso del planeta, por lo cual recibe el nombre de archipiélago patagónico austral. Latitudinalmente a lo largo de la costa del Pacífico sur oriental, se pueden reconocer dos grupos faunísticos, uno al norte de los $42^{\circ} \mathrm{S}$ perteneciente a la región cálido temperada y uno al sur de los $42^{\circ} \mathrm{S}$ perteneciente a la región frío temperada (Balech 1954, Ojeda et al. 2000). Toda la costa del Pacífico sur oriental presenta tres regiones batimétricas con implicancias biogeográficas para la fauna íctica: una plataforma continental que se extiende desde la línea de mareas hasta los $200 \mathrm{~m}$ de profundidad, un talud continental superior que se extiende desde los $200 \mathrm{~m}$ hasta los $1.000 \mathrm{~m}$, y un talud continental inferior que se extiende desde los 1.000 $\mathrm{m}$ a los 3.000 m, aproximadamente (Pequeño 2000a).
En este vasto sector, los estudios en condrictios se han centrado al norte del paralelo $42^{\circ} \mathrm{S}$, sobre la plataforma y talud continental (De Buen 1960, Yáñez \& Barbieri 1974, Meléndez 1984, Meléndez \& Meneses 1989, Pequeño 1989, Pequeño et al. 1991, Sielfeld \& Vargas 1992, Sielfeld \& Vargas 1996, Bearez 1996, Long 1997, Pequeño 1997, Chirichigno \& Vélez 1998, Brito 2004). En tanto que, al sur del paralelo $42^{\circ} \mathrm{S}$ el conocimiento de la distribución geográfica y hábitos de casi todas las especies de Chondrichthyes presenta limitaciones, particularmente de aquellas que habitan aguas profundas (Lamilla 2005) debido a que los pocos estudios realizados en aguas patagónicas se han centrado al interior de los canales y fiordos (Guzmán \& Campodónico 1976, Guzmán 1978, Lloris \& Rucabado 1991, Matallanas et al. 1993) o en la angosta plataforma continental sur patagónica y el talud continental superior (Norman 1937, Ojeda 1983, Pequeño \& Lamilla 1985, Lamilla 1986, Pequeño \& Lamilla 1996, Lamilla \& Pequeño 1999, Pequeño 2000b). 
La ausencia de investigaciones científicas en aguas profundas del archipiélago patagónico austral ha generado que dicho sector actualmente sea una de las áreas con mayor déficit de conocimiento ictiológico del planeta (Pequeño 2000a), particularmente con respecto a su fauna de Chondrichthyes, pues no existe información disponible (Compagno 1984a y b, Sielfeld \& Vargas 1999). Esta situación genera incertidumbre científica debido a que los peces cartilaginosos de aguas profundas del extremo sur patagónico, islas Diego Ramírez y Paso Drake son los condrictios no antárticos más australes del planeta, cuya importancia biogeográfica es de insospechado valor científico, especialmente por ser este lugar el punto de unión de dos océanos.

En este contexto, el objetivo del presente trabajo fue describir la diversidad, distribución, riqueza y abundancia de condrictios del talud continental del extremo austral de la costa Pacífica sudamericana, para mejorar el conocimiento acerca de los Chondrichthyes de aguas profundas del Pacífico sur, Atlántico sur y océano austral.

\section{Material y métodos}

El área de estudio comprendió el sector de operaciones de la flota industrial chilena de palangre dedicada a la extracción del recurso bacalao de profundidad (Dissostichus eleginoides, Smitt 1898), entre las latitudes 5243’S (Faro Evangelistas, boca occidental del Estrecho de Magallanes) y 5705'S (Paso Drake, al sur de las islas Diego Ramírez) y las longitudes 6548’W (bahía Nassau, en el límite marítimo de Chile y Argentina) y $75^{\circ} 46^{\prime} \mathrm{W}$ (isla Decepción) (Fig. 1). Las profundidades de estudio estuvieron entre los $168 \mathrm{~m}$ y $2.250 \mathrm{~m}$, con el $97 \%$ de las estaciones de estudio desarrolladas a más de $1.000 \mathrm{~m}$ de profundidad.

Se analizaron 107 estaciones de muestreo distribuidas homogéneamente a través del área de estudio, durante la primavera y el verano temprano de 2007 (10 de septiembre a 22 de diciembre). En cada estación se registró la captura total de condrictios, en número de ejemplares y en biomasa. El número de ejemplares se determinó por conteo directo en la factoría del buque, donde los especimenes eran mantenidos hasta que finalizara el trabajo de virado del palangre de cada estación, para ser descartados posteriormente al mar. La biomasa fue determinada por pesaje directo de cada espécimen capturado en la factoría del buque, excepto los dos especimenes de Somniosus pacificus, Bigelow \& Schroeder 1944, los que debido a su tamaño no pudieron ser embarcados, en este caso se estimó su longitud y peso. Finalmente, la longitud total de los especimenes se registró por medición directa empleando una cinta métrica. Los especimenes fueron identificados taxonómicamente siguiendo a Bizikov et al. (2004), Compagno (1984a), Cousseau et al. (2000), Díaz de Astarloa \& Mabragaña (2004), Lamilla \& Sáez (2003) y Yano et al. (2004). Dada las dificultades logísticas inherentes al trabajo efectuado a bordo de un buque pesquero, no fue posible conservar los ejemplares para remitirlos a alguna colección zoológica.

Se determinó la diversidad del ensamble de condrictios a través del índice de Shannon-Weaver. Este índice expresa la uniformidad de los valores de importancia a través de todas las especies de la muestra (Peet 1974, Magurran 1988). Adquiere valores entre cero, cuando hay una sola especie, y el logaritmo de S, cuando todas las especies están representadas por el mismo número de individuos (Magurran 1988). Este cálculo se realizó en base a todos los especimenes presentes en las estaciones muestreadas, según la expresión:

$$
H^{\top}(\text { bits } / \text { ind })=-\sum_{i=1}^{S} p_{i} * \log _{2} p_{i}
$$

donde $\mathrm{s}=$ número de especies, $p_{i}=$ proporción de la especie i en la muestra

Además, se empleó el índice de equidad de Pielou para medir la proporción de la diversidad observada en cada estación con relación a la máxima diversidad esperada. Su valor va de 0 a 1 , de forma que 1 corresponde a situaciones donde todas las especies son igualmente abundantes (Magurran 1988). El cálculo se efectuó según la siguiente expresión:

$$
J^{\prime}=\frac{H^{\prime}}{H^{\prime}{ }_{\max }}
$$

donde $\mathrm{H}_{\text {max }}{ }^{\prime}=\ln (\mathrm{S})$ y $\mathrm{H}^{\prime}=$ medida logarítmica de la diversidad.

Finalmente, se empleó el Coeficiente de Similitud de Jaccard para expresar el grado en el que dos muestras son semejantes por las especies presentes en ellas, por lo que son una medida inversa de la diversidad, que se refiere al cambio de especies entre dos estaciones (Pielou 1975, Magurran 1988). El intervalo de valores para el índice de Jaccard va de 0 , cuando no hay especies compartidas entre ambas estaciones, hasta 1, cuando dos estaciones tienen la misma composición de especies. Este coeficiente se obtuvo según la siguiente expresión:

$$
I_{j}=\frac{c}{a+b-c}
$$

donde $\mathrm{a}=$ número de especies presentes en el sitio $\mathrm{A}$, $\mathrm{b}=$ número de especies presentes en el sitio $\mathrm{B}$ y $\mathrm{c}=$ número de especies presentes en ambos sitios $\mathrm{A}$ y $\mathrm{B}$. 


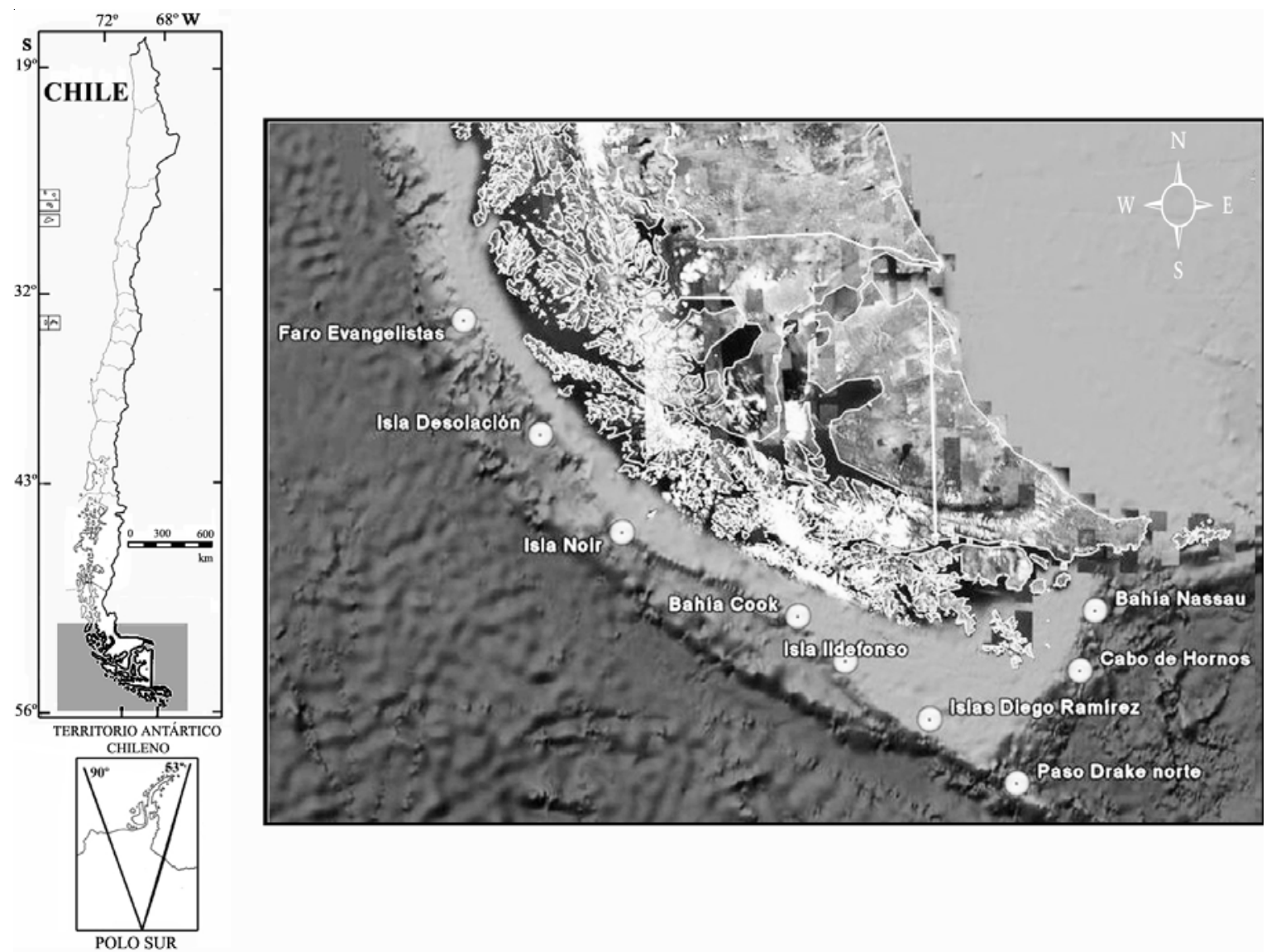

Figura 1

Estaciones de muestreo en el archipiélago patagónico austral, Cabo de Hornos, islas Diego Ramírez y el sector norte del paso Drake. Google Earth, fecha de captura 01 de febrero 2009

Sampling station locations for the south Patagonian Archipelago, Cape Horn, Diego Ramirez islands and the northern area of the Drake Passage. Google Earth, visited February 012009

\section{Resultados}

Se encontraron siete especies de condrictios distribuidos en los ordenes Rajiformes y Squaliformes, representadas en dos familias cada uno: Arhynchobatidae y Rajidae, Squalidae y Somniosidae respectivamente para Rajiformes y Squaliformes, mientras que el orden Lamniformes sólo presentó una familia: Lamnidae, con una especie.

Cuatro especies correspondieron a batoideos; Amblyraja frerichsi (Krefft, 1968), Bathyraja cousseauae Díaz de Astarloa \& Mabragaña, 2004, Bathyraja meridionalis Stehmann, 1987 y Bathyraja macloviana (Norman, 1937) y tres correspondieron a tiburones; Somniosus pacificus Bigelow \& Schroeder, 1944, Squalus acanthias Linnaeus, 1758 y Lamna nasus (Bonnaterre, 1788). Si bien ninguna de las especies señaladas había sido registrada previamente en el área y profundidad específicas del estudio (Tabla 1), todas ellas lo han sido anteriormente en las periferias o en aguas someras de la Patagonia (Argentina, Uruguay, Islas Malvinas, Chile central) (Compagno 1984a, b, Pequeño \& Lamilla 1985, Pequeño 1989, Lloris \& Rucabado 1991, Menni \& Stehmann 2000, Nion et al. 2002, Lamilla \& Sáez 2003).

Aunque la distribución de las especies no es homogénea, Amblyraja frerichsi fue la única capturada en toda el área de estudio (Tabla 2). Bathyraja cousseauae, especie que nunca había sido reportada en el Pacifico sur oriental, fue capturada en las estaciones australes, al sur del paralelo $56^{\circ} \mathrm{S}$. Bathyraja meridionalis, que al igual que $B$. cousseauae, nunca había sido reportada en el Pacifico sur oriental, y B. macloviana fueron

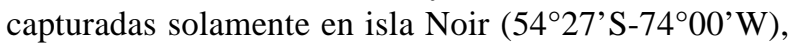
lugar en el cual además fueron capturados los dos únicos 


\section{Tabla 1}

Distribución geográfica conocida en el océano Pacífico sur y aguas adyacentes de las especies de condrictios halladas en aguas del talud continental profundo del extremo sur patagónico de la costa Pacífica sudamericana, islas Diego Ramírez y paso Drake

Geographical distribution in the southeastern Pacific Ocean and adjacent areas of the Chondrichthyan species found in deep waters of southern Patagonian, Diego Ramirez islands and Drake passage

\begin{tabular}{|c|c|c|c|c|c|}
\hline Especie & $\begin{array}{l}\text { Distribución } \\
\text { latitudinal en el } \\
\text { Pacifico sur } \\
\text { oriental }\end{array}$ & $\begin{array}{c}\text { Distribución } \\
\text { longitudinal en el } \\
\text { Pacifico sur } \\
\text { oriental }\end{array}$ & $\begin{array}{l}\text { Distribución } \\
\text { batimétrica en el } \\
\text { Pacífico sur }\end{array}$ & $\begin{array}{l}\text { Presencia en el } \\
\text { Atlántico sur } \\
\text { occidental }\end{array}$ & $\begin{array}{l}\text { Presencia en el } \\
\text { océano Austral }\end{array}$ \\
\hline Amblyraja frerichsi & $\begin{array}{l}\text { desde } 21^{\circ} 25^{\prime} \text { 'S } \\
\text { hasta } 49^{\circ} 01 \text { 's }\end{array}$ & $\begin{array}{l}\text { desde } 70^{\circ} 33^{\prime} \mathrm{W} \\
\text { hasta } 76^{\circ} 18^{\prime} \mathrm{W}\end{array}$ & $600-2.000 \mathrm{~m}$ & $\begin{array}{l}\text { Argentina, } \\
\text { Malvinas, } \\
\text { Uruguay }\end{array}$ & No \\
\hline Bathyraja meridionalis & $\begin{array}{c}\text { Sin registros } \\
\text { previos }\end{array}$ & $\begin{array}{c}\text { Sin registros } \\
\text { previos }\end{array}$ & $\begin{array}{l}\text { Sin registros } \\
\text { previos }\end{array}$ & $\begin{array}{l}\text { Argentina, } \\
\text { Malvinas, } \\
\text { Uruguay }\end{array}$ & $\mathrm{Si}$ \\
\hline Bathyraja cousseauae & $\begin{array}{c}\text { Sin registros } \\
\text { previos }\end{array}$ & $\begin{array}{l}\text { Sin registros } \\
\text { previos }\end{array}$ & $\begin{array}{c}\text { Sin registros } \\
\text { previos }\end{array}$ & $\begin{array}{l}\text { Argentina, } \\
\text { Malvinas, } \\
\text { Uruguay }\end{array}$ & No \\
\hline Bathyraja macloviana & $\begin{array}{l}\text { desde } 51^{\circ} 00^{\prime} \mathrm{S} \\
\text { hasta } 54^{\circ} 55^{\prime} \mathrm{S}\end{array}$ & $\begin{array}{l}\text { desde } 67^{\circ} 09^{\prime} \mathrm{W} \\
\text { hasta } 75^{\circ} 44^{\prime} \mathrm{W}\end{array}$ & $122-300 \mathrm{~m}$ & $\begin{array}{c}\text { Argentina, } \\
\text { Malvinas, } \\
\text { Uruguay }\end{array}$ & No \\
\hline Somniosus pacificus & $\begin{array}{l}\text { desde } 13^{\circ} 42^{\prime} \mathrm{S} \\
\text { hasta } 54^{\circ} 55^{\prime} \mathrm{S}\end{array}$ & $\begin{array}{l}\text { desde } 153^{\circ} \mathrm{E} \\
\text { hasta } 72^{\circ} \mathrm{W}\end{array}$ & $0-2000 \mathrm{~m}$ & $\begin{array}{c}\text { Argentina, } \\
\text { Malvinas, } \\
\text { Uruguay, Brasil }\end{array}$ & $\mathrm{Si}$ \\
\hline Squalus acanthias & $\begin{array}{l}\text { desde } 00^{\circ} 00^{\prime} \mathrm{S} \\
\text { hasta } 54^{\circ} 55 \text { 'S }\end{array}$ & $\begin{array}{l}\text { desde } 81^{\circ} 25^{\prime} \mathrm{W} \\
\text { hasta } 67^{\circ} 09^{\prime} \mathrm{W}\end{array}$ & $0-1460 \mathrm{~m}$ & $\begin{array}{c}\text { Argentina, } \\
\text { Malvinas, } \\
\text { Uruguay, Brasil }\end{array}$ & No \\
\hline Lamna nasus & $\begin{array}{l}\text { desde } 18^{\circ} 20^{\prime} \mathrm{S} \\
\text { hasta } 54^{\circ} 55^{\prime} \mathrm{S}\end{array}$ & $\begin{array}{c}\text { desde } 153^{\circ} \mathrm{E} \\
\text { hasta } 65^{\circ} 42^{\prime} \mathrm{W}\end{array}$ & $0-740 \mathrm{~m}$ & $\begin{array}{c}\text { Argentina, } \\
\text { Malvinas, } \\
\text { Uruguay, Brasil }\end{array}$ & $\mathrm{Si}$ \\
\hline
\end{tabular}

\section{Tabla 2}

Antecedentes de los ejemplares capturados en el área de estudio durante esta investigación. Los especimenes fueron estudiados por especie. Sexo = número de especimenes por sexo; LT (cm) = rango de longitud total (longitud total mínimamáxima); Biomasa (kg) = biomasa total de la especie por sexo; Área = área donde se capturaron condrictios; Lat / Long =

latitudes y longitudes que delimitan el área de registro; Profundidad $(\mathrm{m})$ = rango de profundidad de las estaciones con presencia. ND = información no disponible

Data of the specimens caugth on the studied area during this research. The specimens where studied by species. Sex $=$ number of specimens by sex; LT $(\mathrm{cm})=$ total length range (smallest length-biggest length) $(\mathrm{TL})$; Biomass $(\mathrm{kg})=$ total biomass by species and sex; Area = chondrichthyan capture area; Lat / Long = latitudes and longitudes delimiting area with records; Depth $(\mathrm{m})=$ depth range with presence. $\mathrm{ND}=$ information not available

\begin{tabular}{|c|c|c|c|c|c|c|}
\hline Especie & Sexo & $\mathrm{LT}(\mathrm{cm})$ & Biomasa (kg) & Área & Lat $\left({ }^{\circ} \mathrm{S}\right) /$ Long $\left({ }^{\circ} \mathrm{W}\right)$ & Profundidad (m) \\
\hline A. frerichsi & $\begin{array}{l}\delta(n=197) \\
\text { o }(n=155)\end{array}$ & $\begin{array}{l}79-120 \\
55-132\end{array}$ & $\begin{array}{l}1735,0 \\
1365,0\end{array}$ & Toda el área & $\begin{array}{l}52^{\circ} 43^{\prime} \mathrm{S}-57^{\circ} 05^{\prime} \mathrm{S} / \\
65^{\circ} 48^{\prime} \mathrm{W}-75^{\circ} 46^{\prime} \mathrm{W}\end{array}$ & $168-2250$ \\
\hline B. meridionalis & $\delta^{*}$ & 144 & 15,0 & Isla Noir & $54^{\circ} 27^{\prime} \mathrm{S} / 74^{\circ} 00^{\prime} \mathrm{W}$ & $952-1200$ \\
\hline B. cousseauae & o & $\begin{array}{c}98 \\
111\end{array}$ & $\begin{array}{c}7,5 \\
12,5\end{array}$ & $\begin{array}{l}\text { Islas Diego Ramírez, } \\
\text { Cabo de Hornos }\end{array}$ & $\begin{array}{c}56^{\circ} 39^{\prime} \mathrm{S}-56^{\circ} 14^{\prime} \mathrm{S} / \\
68^{\circ} 57^{\prime} \mathrm{W} \\
-66^{\circ} 33^{\prime} \mathrm{W}\end{array}$ & $481-1192$ \\
\hline B. macloviana & $\delta^{*}$ & 98 & 3,0 & Islas Diego Ramírez & $56^{\circ} 39^{\prime} \mathrm{S} / 68^{\circ} 57^{\prime} \mathrm{W}$ & $631-956$ \\
\hline S. pacificus & $\begin{array}{l}\text { ND } \\
\text { ND }\end{array}$ & $\begin{array}{l} \pm 400 \\
\pm 400\end{array}$ & $\begin{array}{l} \pm 1000,0 \\
\pm 1000,0\end{array}$ & Isla Noir & $\begin{array}{l}54^{\circ} 35^{\prime} \mathrm{S}-54^{\circ} 33^{\prime} \mathrm{S} / \\
73^{\circ} 58^{\prime} \mathrm{W}-74^{\circ} 05^{\prime} \mathrm{W}\end{array}$ & $1230-1549$ \\
\hline S. acanthias & 옹 & $\begin{array}{l}77 \\
52\end{array}$ & $\begin{array}{l}3,8 \\
2,2\end{array}$ & $\begin{array}{l}\text { Isla Diego Ramírez, } \\
\text { Cabo de Hornos }\end{array}$ & $\begin{array}{l}56^{\circ} 01^{\prime} \mathrm{S}-56^{\circ} 26^{\prime} \mathrm{S} / \\
66^{\circ} 01^{\prime} \mathrm{W}-69^{\circ} 30^{\prime} \mathrm{W}\end{array}$ & $168-1978$ \\
\hline L. nasus & $\delta^{*}$ & 190 & 130,0 & Isla Desolación & $53^{\circ} 07^{\prime} \mathrm{S} / 75^{\circ} 12^{\prime} \mathrm{W}$ & $1301-1809$ \\
\hline
\end{tabular}


Tabla 3

Análisis de la participación, por nivel taxonómico, a la diversidad, biomasa y abundancia de la fauna de condrictios presente en aguas profundas del extremo sur patagónico la costa del océano Pacifico sur oriental

Diversity, abundance and biomass analysis of participation, by taxonomic level, of Chondrichthyan fauna present in deep waters of the southern Patagonian coast of the south eastern Pacific Ocean

\begin{tabular}{|c|c|c|c|c|c|c|c|c|}
\hline Orden & $\begin{array}{c}\text { Participación del } \\
\text { Orden en la } \\
\text { diversidad } \\
\%\end{array}$ & $\begin{array}{c}\text { Participación del } \\
\text { Orden en la } \\
\text { biomasa } \\
\%\end{array}$ & Familia & $\begin{array}{c}\text { Participación de la } \\
\text { familia en la } \\
\text { diversidad } \\
\%\end{array}$ & $\begin{array}{c}\text { Participación de la } \\
\text { familia en la } \\
\text { biomasa } \\
\%\end{array}$ & Especie & $\begin{array}{c}\text { Participación de } \\
\text { la especie en la } \\
\text { diversidad } \\
\%\end{array}$ & $\begin{array}{c}\text { Participación de } \\
\text { la especie en la } \\
\text { abundancia (n) } \\
\%\end{array}$ \\
\hline \multirow[t]{2}{*}{ Squaliformes } & 28,57 & 38,04 & Squalidae & 14,29 & 0,11 & Squalus acanthias & 0,11 & 0,55 \\
\hline & & & Somniosidae & 14,29 & 37,92 & Somniosus pacificus & 37,92 & 0,55 \\
\hline Lamniformes & 14,29 & 2,46 & Lamnidae & 14,29 & 2,46 & Lamna nasus & 2,46 & 0,28 \\
\hline \multirow[t]{4}{*}{ Rajiformes } & 57,14 & 59,5 & Rajidae & 14,29 & 58,78 & Amblyraja frerichsi & 58,78 & 97,51 \\
\hline & & & Arhynchobatidae & 42,86 & 0,72 & Bathyraja cousseauae & 0,38 & 0,55 \\
\hline & & & & & & Bathyraja meridionalis & 0,28 & 0,28 \\
\hline & & & & & & Bathyraja macloviana & 0,06 & 0,28 \\
\hline
\end{tabular}

especimenes de Somniosus pacificus. Squalus acanthias fue otra especie capturada sólo en el sector austral, al sur de los $56^{\circ} \mathrm{S}$, en los que posiblemente constituyen los registros más australes para esta especie. Lamna nasus, en tanto, fue capturada en una de las estaciones septentrionales, en isla Desolación $\left(53^{\circ} \mathrm{S}\right)$ cerca de la boca occidental del estrecho de Magallanes. La mayor amplitud de distribución batimétrica fue observada en las especies Amblyraja frerichsi y S. acanthias (Tabla 2).

La familia con mayor riqueza de especies en el sector, fue Arhynchobatidae ('batoideos') con tres especies, lo que representa casi la mitad del total de especies registradas, seguida por Rajidae, Lamnidae, Squalidae y Somniosidae, cada una representada por una especie.

En el área de estudio la biomasa capturada de Chondrichthyes fue de $5.346 \mathrm{~kg}$, siendo la familia Rajidae la que presentó la mayor biomasa con $3.142 \mathrm{~kg}(58,8 \%)$, seguida por Somniosidae con $2.027 \mathrm{~kg}$ (37,9\%) la cual se destaca pues tal porcentaje se debe a sólo dos especimenes capturados. Las restantes familias se encontraron presentes con bajos aportes a la biomasa total (Tabla 2).

La abundancia de especimenes por familia estuvo dominada por Rajidae con 352 ejemplares, lo que representa el 97,5\% del total de ejemplares capturados, seguida por Arhynchobatidae con 4 ejemplares, lo que representa el 1,1\% de los ejemplares totales. Las otras familias son poco abundantes en el área (Tabla 3).

La abundancia total de especimenes presentó máximos en las cercanías de las islas Diego Ramírez y mínimos en las cercanías de Faro Evangelistas, en el sector boreal del área de estudios. La riqueza de especies también presentó su máximo en las islas Diego Ramírez, donde se observaron 4 especies, presentando la mayoría de las estaciones una especie. Al contrario de la riqueza y la abundancia, que se concentran en el área sur, la diversidad lo hace en el área norte, en Isla Desolación con 0,21 bits ind..$^{-1}$, llegando a 0 bits ind. ${ }^{-1}$ en las estaciones que presentaron solo una especie. La equidad presentó valores similares a los de diversidad, con un máximo de 0,24 en isla Desolación y mínimos de 0,0 en cinco estaciones (Tabla 4). El índice de similitud de Jaccard indica que no hay estaciones con cero similitud, lo cual significa que todas las estaciones del archipiélago patagónico austral, Cabo de Hornos, islas Diego Ramírez y el sector norte del paso Drake comparten al menos una especie de condrictios. A su vez, diversas estaciones que presentan similitud 1,0 lo cual indica similitud total de especies (Tabla 5).

\section{Discusión}

Hasta la fecha el escaso conocimiento de los Chondrichthyes del talud continental profundo de la zona austral del Pacífico sur oriental indicaba sólo la presencia de la familia Squalidae (Pequeño 2000). Con la presente investigación se confirma la presencia de Squalidae y agrega la presencia de las familias Arhynchobatidae, Rajidae, Lamnidae y Somniosidae, lo que permite caracterizar al talud continental profundo del archipiélago patagónico austral como un lugar en el cual los Chondrichthyes pertenecientes a diferentes grupos biogeográficos podrían estar confluyendo debido al contacto del océano Atlántico y Pacífico y a la cercanía geográfica del océano Austral.

La fauna de condrictios detectada en aguas profundas de la zona austral del Pacífico sur oriental presenta una fuerte similitud con la presente en aguas del Atlántico sur 


\section{Tabla 4}

\section{Abundancia total (n individuos), Riqueza (n especies), índice de diversidad de Shannon-Weaver (H') e índice de equidad de Pielou $\left(\mathrm{J}^{\prime}\right)$ de especies de condrictios, a través del archipiélago patagónico austral, Cabo de Hornos, islas Diego Ramírez y el sector norte del paso Drake}

Total abundance (n specimens), richness (n species), diversity index of Shannon-Weaver (H') and Pielou equitability index (J') of Chondrichthyan species, through the southern Patagonian Archipelago, Cape Horn,

Diego Ramirez Islands and the northern sector of the Drake passage

\begin{tabular}{|c|c|c|c|c|c|c|c|c|c|}
\hline \multirow[b]{2}{*}{ Especie } & \multirow[b]{2}{*}{ Paso Drake } & \multirow[b]{2}{*}{$\begin{array}{c}\text { Diego } \\
\text { Ramírez }\end{array}$} & \multirow[b]{2}{*}{$\begin{array}{l}\text { Cabo de } \\
\text { Hornos }\end{array}$} & \multirow[b]{2}{*}{$\begin{array}{c}\text { Bahía } \\
\text { Nassau }\end{array}$} & \multicolumn{2}{|c|}{ Estación } & \multirow[b]{2}{*}{ Isla Noir } & \multirow[b]{2}{*}{$\begin{array}{c}\text { Isla } \\
\text { Desolación }\end{array}$} & \multirow[b]{2}{*}{$\begin{array}{c}\text { Faro } \\
\text { Evangelistas }\end{array}$} \\
\hline & & & & & $\begin{array}{c}\text { Isla } \\
\text { Ildefonso }\end{array}$ & $\begin{array}{l}\text { Bahía } \\
\text { Cook }\end{array}$ & & & \\
\hline A. frerichsi & 17 & 92 & 39 & 67 & 37 & 24 & 68 & 6 & 2 \\
\hline B. cousseauae & - & 1 & 1 & - & - & - & - & - & - \\
\hline B. meridionalis & - & - & - & - & - & - & 1 & - & - \\
\hline B. macloviana & - & 1 & - & - & - & - & & - & - \\
\hline S. pacificus & - & - & - & - & - & - & 2 & - & - \\
\hline S. acanthias & - & 1 & 1 & - & - & - & - & - & - \\
\hline L. nasus & - & - & - & - & - & - & - & 1 & - \\
\hline Abundancia total & 17 & 95 & 41 & 67 & 37 & 24 & 71 & 7 & 2 \\
\hline Riqueza & 1 & 4 & 3 & 1 & 1 & 1 & 3 & 2 & 1 \\
\hline Diversidad & 0,00 & 0,09 & 0,12 & 0,00 & 0,00 & 0,00 & 0,10 & 0,21 & 0,00 \\
\hline Equidad & 0,00 & 0,10 & 0,13 & 0,00 & 0,00 & 0,00 & 0,12 & 0,24 & 0,00 \\
\hline
\end{tabular}

occidental, más incluso que con la existente en latitudes medias o bajas del Pacífico sur oriental. Todas las especies capturadas en el sector han sido registradas previamente en Islas Malvinas, Argentina y Uruguay. En la costa continental chilena al norte del paralelo $42^{\circ} \mathrm{S}$ (límite estructural del archipiélago patagónico austral) sólo ha sido registrado previamente el 57,1\% de las especies registradas en las aguas patagónicas profundas y sólo un 28,6\% de las especies ha sido detectada en latitudes bajas del Pacífico sur oriental, en Perú. Finalmente, el 42,9\% de las especies detectadas en el presente estudio se encuentran en aguas antárticas. Así, la fuerte relación entre la fauna de condrictios de aguas profundas de la zona austral del Pacífico sur oriental con la del Atlántico sur occidental corrobora los patrones biogeográficos observados para peces costeros en investigaciones recientes; Pequeño (1999), tras analizar los peces litorales de los fiordos chilenos, sugiere que la zona austral del Pacifico sur oriental puede ser reconocida como una sola unidad ictiogeográfica con aguas del Atlántico sur occidental, lo cual fue corroborado por Ojeda et al. (2000) quienes asignan un grado de similitud de un 85,0\%, para la fauna de peces costeros de ambos sectores.

La abundancia de especimenes (n de individuos) presentó valores máximos en las islas Diego Ramírez, determinada en gran medida por la abundancia de Amblyraja frerichsi, especie que representa el 97,51\% de la abundancia total de condrictios en el área (Tabla 3). Los mínimos fueron observados en las cercanías de Faro
Evangelistas, en el sector boreal del área de estudios, lo cual más que indicar diferencias latitudinales en la abundancia de condrictios, refleja el heterogéneo número de muestreos, reducidos en el área norte (Tabla 4). El máximo en la riqueza de especies fue observado también en Diego Ramírez (4 especies), los mínimos se observaron en el área central del estudio, donde solo se presentó $A$. frerichsi. Aunque la riqueza y la abundancia se centraron en el área sur, la diversidad fue máxima en el área norte (isla Desolación) con 0.21 bits ind. ${ }^{-1}$. Las estaciones que presentaron solo una especie presentaron 0 bits ind. ${ }^{-1}$. La equidad presentó un comportamiento similar al de la diversidad (Tabla 4). La similitud total (índice de Jaccard $=1,0$ ) observada entre varias estaciones del sector se explica por la presencia de $A$. frerichsi, especie distribuida en todo el área de estudio, y en determinadas estaciones presente como especie única. La misma especie es la que explica la ausencia de de estaciones con disimilitud total (índice de Jaccard $=0,0$ ), al encontrarse presente en todas ellas (Tabla 5).

La riqueza y biomasa de los batoideos de aguas profundas registrada en el área de estudio no se observa en latitudes medias y bajas del Pacífico sur oriental (norte del paralelo $42^{\circ}$ S) donde la biomasa y riqueza de especies de condrictios del talud continental se encuentran dominadas por los tiburones, especialmente del orden Squaliformes (Meléndez \& Meneses 1989, Ojeda et al. 2000, Sielfeld \& Vargas 1996). Este predominio de tiburones en bajas latitudes y de rayas en altas latitudes 
Tabla 5

Coeficiente de similitud de Jaccard (J) de especies de condrictios, a través del archipiélago patagónico austral, Cabo de Hornos, Islas Diego Ramírez y el sector norte del paso Drake

Jaccard similarity coefficient (J) of Chondrichthyan species, through the southern Patagonian Archipelago, Cape Horn, Diego Ramirez Islands and the northern sector of the Drake passage

\begin{tabular}{lccccccccc}
\hline \multicolumn{1}{c}{ Estación } & $\begin{array}{c}\text { Paso } \\
\text { Drake }\end{array}$ & $\begin{array}{c}\text { Diego } \\
\text { Ramírez }\end{array}$ & $\begin{array}{c}\text { Cabo de } \\
\text { Hornos }\end{array}$ & $\begin{array}{c}\text { Bahía } \\
\text { Nassau }\end{array}$ & $\begin{array}{c}\text { Isla } \\
\text { Ildefonso }\end{array}$ & $\begin{array}{c}\text { Bahía } \\
\text { Cook }\end{array}$ & $\begin{array}{c}\text { Isla } \\
\text { Noir }\end{array}$ & $\begin{array}{c}\text { Isla } \\
\text { Desolación }\end{array}$ & $\begin{array}{c}\text { Faro } \\
\text { Evangelistas }\end{array}$ \\
\hline Paso Drake & - & - & - & - & - & - & - & - & - \\
Diego Ramírez & 0,25 & - & - & - & - & - & - & - & - \\
Cabo de Hornos & 0,33 & 0,75 & - & - & - & - & - & - & - \\
Bahía Nassau & 1,00 & 0,25 & 0,33 & - & - & - & - & - & - \\
Isla Ildefonso & 1,00 & 0,25 & 0,33 & 1,00 & - & - & - & - & - \\
Bahía Cook & 1,00 & 0,25 & 0,33 & 1,00 & 1,00 & - & - & - & - \\
Isla Noir & 0,33 & 0,17 & 0,20 & 0,33 & 0,33 & 0,33 & - & - & - \\
Isla Desolación & 0,50 & 0,20 & 0,25 & 0,50 & 0,50 & 0,50 & 0,25 & - & - \\
Faro Evangelistas & 1,00 & 0,25 & 0,33 & 1,00 & 1,00 & 1,00 & 0,33 & 0,50 & - \\
\hline
\end{tabular}

implica que en el Pacífico sur oriental existe un quiebre latitudinal en la riqueza de especies y abundancia.

Es importante resaltar la biomasa capturada de Chondrichthyes que se registró en el presente estudio, debido a que, al mismo tiempo, en el sector operaron otros 9 buques con el mismo sistema de pesca (Moreno et al. 2008), lo que llevaría a pensar que la captura y descarte total de Chondrichthyes en el área durante el periodo podría ser del orden de $48.114 \mathrm{~kg}$. Esta captura teórica se considera elevada por la vulnerabilidad que presentan los condrictios de aguas profundas (Kyne \& Simpfendorfer 2007).

Un mayor número de estudios se deben llevar a cabo con el fin de conocer los factores que estarían influyendo sobre la presencia de las especies en esta área del océano Pacifico austral.

\section{Agradecimientos}

En nombre de la tripulación del buque palangrero Isla Santa Clara, agradecemos al Sr. Ramón Ferreiras, Patrón de Pesca, por las facilidades otorgadas abordo. Al Dr. Carlos Moreno del Instituto de Ecología y Evolución de la Universidad Austral de Chile, por proveer la logística del trabajo en terreno. Al Dr. Matthias Stehmann del Ichthyological Research Laboratory de Hamburg, Alemania, por identificar taxonómicamente a los ejemplares de $B$. cousseauae analizados y corroborar la identificación de B. meridionales. Al Sr. Joost Pompert del Falkland Islands Fisheries Department, por sus aportes bibliográficos. JP Torres-Florez agradece el financiamiento recibido a través de una beca doctoral de
CONICYT-Chile. Finalmente, a los evaluadores anónimos por sus sugerencias y comentarios.

\section{Literatura citada}

Balech E. 1954. División zoogeográfica del litoral sudamericano. Revista de Biología Marina 4: 184-195.

Bearez P. 1996. Lista de los peces marinos del Ecuador continental. Revista de Biología Tropical 44(2): 731-74.

Bizilov V, AArkhipkin, V Laptikhovsky \& J Pompert. 2004. Identification guide and biology of the Falkland skates, 36 pp. Fisheries Department, Falkland Island Government, Stanley.

Brito J. 2004. Hallazgo de Somniosus pacificus Bigelow \& Schroeder, 1944 (Squaliformes: Squalidae) en San Antonio, Chile central. Investigaciones Marinas 32(2): 137-139.

Chirichigno N \& J Vélez. 1998. Clave para la identificación de los peces marinos del Perú, 496 pp. Instituto del Mar del Perú, Callao.

Compagno L. 1984a. FAO species catalogue. Vol. 4. Sharks of the world. An annotated and illustrated catalogue of shark species known to date. Part 1. Hexanchiformes to Lamniformes. FAO Fisheries Synopsis 125 4(1): 1-249.

Compagno L. 1984b. FAO species catalogue. Vol. 4. Sharks of the world. An annotated and illustrated catalogue of shark species known to date. Part 2. Carcharhiniformes. FAO Fisheries Synopsis 125 4(2): 1-655.

Compagno L. 2001. Sharks of the world. An annotated and illustrated catalogue of shark species known to date. Vol. 2. Bullhead, mackerel and carpet sharks (Heterodontiformes, Lamniformes and Orectolobiformes). FAO Species Catalogue for Fishery Purposes 1(2): 1-269. 
Cousseau M, D Figueroa \& J Díaz de Astarloa. 2000. Clave de identificación de las rayas del litoral marítimo de Argentina y Uruguay (Chondrichthyes, Familia Rajidae). Contribución INIDEP 1079: 5-35.

De Buen F. 1960. Tiburones, rayas y quimeras de la Estación de Biología Marina de Montemar, Chile. Revista de Biología Marina 10(1-3): 1-50.

Díaz de Astarloa J \& E Mabragaña. 2004. Bathyraja cousseauae sp. n.: a new softnose skate from the southwestern Atlantic (Rajiformes, Rajidae). Copeia (2): 326-335.

Guzmán L. 1978. Registro de Cetorhinus maximus (Gunner, 1765) en aguas de Chile austral (Elasmobranchii: Cetorhinidae). Anales del Instituto de la Patagonia, Serie Ciencias Naturales 9: 228 -230.

Guzmán L \& I Campodónico. 1976. Notorhynchus cepedianus (Peron, 1807) en la entrada oriental de Estrecho de Magallanes. Anales del Instituto de la Patagonia, Serie Ciencias Naturales 7: 207-210.

Kyne P \& C Simpfendorfer. 2007. A collation and summarization of available data on deepwater Chondrichthyans: biodiversity, life history and fisheries, 137 pp. IUCN SSC Shark Specialist Group Report, Queensland.

Lamilla J. 1986. Bathyraja albomaculata (Norman, 1937): Primer registro para las costas de Chile (Chondrichthyes, Rajidae). Boletín de la Sociedad de Biología de Concepción 57: 177-182.

Lamilla J. 2005. Lineamientos básicos para desarrollar el plan de acción nacional de tiburones. Volumen 2: Estado actual de la biología y pesquería de condrictios en Chile. Informe Final. Proyecto FIP 2004-18: 1-210.

Lamilla J \& G Pequeño. 1999. Descripción esquelética comparativa y primer registro en el océano Pacífico de Bathyraja multispinis (Norman, 1937) (Rajiformes; Rajoidei), con referencia a otras especies de Bathyraja. Revista de Biología Marina y Oceanografía 34(2): 281-290.

Lamilla J \& S Sáez. 2003. Clave taxonómica para el reconocimiento de especies de rayas chilenas (Chondrichthyes, Batoidei). Investigaciones Marinas 31(2): 3-16.

Lloris D \& J Rucabado. 1991. Ictiofauna del Canal Beagle (Tierra del Fuego), aspectos ecológicos y análisis biogeográfico. Publicación Especial del Instituto Español de Oceanografía 8: 1-182.

Long J. 1997. First account of the birdbeak dogfish shark, Deania calcea (Chondrichthyes: Squalidae) from the northem coast of Perú. Revista de Biología Tropical 45(2): 1-937.

Magurran A. 1988. Ecological diversity and its measurement, 179 pp. Princeton University Press, New Jersey.
Matallanas J, M Carrasson \& M Casadevall. 1993. Observations on the feeding habits of the marrow mouthed cat shark Schroederichthys bivius (Chondrichthyes, Scyliorhinidae) in the Beagle channel. Cybium 17(1): 55-61.

Meléndez R. 1984. Zameus squamulosus (Günther, 1877) en Chile (Chondrichthyes, Squaliformes, Squalidae). Investigaciones Pesqueras 36: 89-95.

Meléndez R \& D Meneses. 1989. Tiburones del talud continental recolectados entre Arica ( $\left(1^{\circ} 19^{\prime} \mathrm{S}\right)$ e Isla Mocha (38³0’S), Chile. Investigaciones Marinas 17: 3-73.

Menni R \& M Stehmann. 2000. Distribution, environment and biology of batoid fishes off Argentina, Uruguay and Brazil. A review. Revista del Museo Argentino de Ciencias Naturales 2(1): 69-109.

Moreno C, R Castro, L Mujica \& P Reyes. 2008. Significant conservation benefits obtained from the use of a new fishing gear in the chilean patagonian toothfish fishery. CCAMLR Science 15: 79-91

Nion H, C Ríos \& P Meneses. 2002. Peces del Uruguay. Lista sistemática y nombres comunes, 105 pp. DINARA, Montevideo.

Norman J. 1937. Coast fishes. Part 2. The Patagonian region. Discovery Report 16: 1-150.

Ojeda P. 1983. Distribución latitudinal y batimétrica de la ictiofauna demersal del extremo austral de Chile. Revista Chilena de Historia Natural 56: 61-70.

Ojeda P, F Labra \& A Muñoz. 2000. Biogeographic patterns of Chilean littoral fishes. Revista Chilena de Historia Natural 73: 625-641.

Peet R. 1975. Relative diversity indices. Ecology 56: 496-498.

Pequeño G. 1989. Peces de Chile. Lista revisada y comentada. Revista de Biología Marina 24(2): 1-132.

Pequeño G. 1997. Peces de Chile. Lista sistemática revisada y comentada: addendum. Revista de Biología Marina y Oceanografía 32(2): 77-94.

Pequeño G. 1999. Peces del crucero CIMAR-FIORDO 2, a los canales patagónicos de Chile, con consideraciones ictiogeográficas. Ciencia y Tecnología del Mar 22: 165-179.

Pequeño G. 2000a. Delimitaciones y relaciones biogeográficas de los peces del Pacifico suroriental. Estudios Oceanológicos 19: 53-76.

Pequeño G. 2000b. Peces del crucero CIMAR-FIORDO 3, a los canales del Sur de Magallanes (ca. $55^{\circ} \mathrm{S}$ ), Chile. Ciencia y Tecnología del Mar 23: 83-94.

Pequeño G \& J Lamilla. 1985. Estudio sobre una colección de rayas del sur de Chile (Chondrichthyes: Rajidae). Revista de Biología Marina 21(2): 225-271.

Pequeño G \& J Lamilla. 1996. Preliminary reports on the fishes collected by the B.I.C. 'Victor Hensen' in the Magellanic Channels, Chile. Berichte zur Polarforschung 190: 68-106. 
Pequeño G, J Lamilla \& A Crovetto. 1991. Captura de Somniosus cf. pacificus Bigelow y Schroeder, 1944, frente a Valdivia, Chile, con notas sobre su contenido gástrico (Chondrichthyes, Squalidae). Estudios Oceanológicos 10: 117-122.

Pielou E. 1975. Ecological diversity, 165 pp. John Wiley \& Sons, New York.

Sielfeld W \& M Vargas 1992. Tiburones y rayas del Pacífico Sur Oriental, 135 pp. Departamento de Ciencias del Mar, Universidad Arturo Prat, Iquique.

Sielfeld W \& M Vargas. 1996. Composición y estructura de la ictiofauna demersal en la zona norte de Chile. Investigaciones Marinas 24: 3-17.
Sielfeld W \& M Vargas. 1999. Review of marine fish zoogeography of Chilean Patagonia $\left(42^{\circ}-57^{\circ} \mathrm{S}\right)$. Scientia Marina 63(1): 451-463.

Yano K, J Stevens \& L Compagno. 2004. A review of the systematics of the sleeper shark genus Somniosus with redescriptions of Somniosus (Somniosus) antarcticus and Somniosus (Rhinoscymnus) longus (Squaliformes: Somniosidae). Ichthyological Research 51(4): 360-373.

Yáñez E \& M Barbieri. 1974. Distribución y abundancia relativa de recursos disponibles a un arte de arrastre camaronero frente a la costa de Valparaíso. Investigaciones Marinas 5(6): 137-156.

Recibido el 14 de diciembre de 2007 y aceptado el 9 de enero de 2009 